\title{
ENABLING TIME-CRITICAL COMMUNICATIONS IN MEDICAL IoT APPLICATIONS
}

\author{
Dino Mustefa ${ }^{1,2}$, Hossein Fotouhi ${ }^{2}$, Sasikumar Punnekkat $^{2}$ and Detlef Scholle ${ }^{1}$ \\ ${ }^{1}$ Embedded Systems, ALTEN Sweden AB, Stockholm, Sweden \\ ${ }^{2}$ Mälardalen University, Västerås, Sweden \\ https://www.alten.se, https://www.mdh.se
}

\begin{abstract}
Efficient communication is paramount for time-critical applications. Emerging time-critical healthcare applications will require extremely low latency, high reliability, and security guarantees. There are existing and emerging network technologies such as $5 \mathrm{G}$ that could enable efficient communications for these time-critical applications. However, it requires detailed identification of the required Quality of Service (QoS) of the applications and careful selection of the appropriate connectivity technology or combination of technologies to fully realize these time-critical healthcare applications. Network slicing is known as a proposed backbone of $5 \mathrm{G}$ technology that aggregates logical network functions and configurations of parameters to support a particular service. In this paper, we address the QoS requirements of medical IoT applications, with a particular focus on their time-critical nature and show how network slicing could be a key technology for meeting such requirements.
\end{abstract}

\section{KEYWORDS}

Medical IoT, Time-Critical Applications, Cybersecurity, Reliability, Wireless Communication, Software-Defined Networking, Network Function Virtualization, Network Slicing

\section{INTRODUCTION}

As life expectancy continues to rise worldwide, the increase in the elderly population places greater demands on the health care system (Rees et al., 2012). Infectious diseases such as COVID -19 are emerging and leaving greater impacts not only on the health sector and people's lives, but also on the global economy. Among other things, this clearly indicates that more effort and investment is required in the realization of novel and complex healthcare solutions, especially for remote monitoring and treatment applications.

One or more sensors and smart medical devices can communicate with each other to achieve a specific medical application. This interaction between the smart sensors and devices using connectivity technologies forms a Medical Internet of Things (MIoT) (Dimitrov, 2016). There are several emerging MIoT applications due to advanced wearable sensors, smart devices and advanced communication technologies such as emerging 5G mobile technology. This will bring many benefits in the field of healthcare. It will enable location-based monitoring and treatment of patients, which will minimize the time required to diagnose and treat diseases and the need for hospitalization and emergency room visits. Moreover, these applications will minimize the transmission of infectious diseases through ubiquitous MIoT infrastructure (Haghi et al., 2020). In general, it will be possible to improve survival rates, especially for patients living in rural areas, and reduce healthcare costs for both patients and healthcare organizations.

By definition, "time-critical applications are those applications whose failure could result in loss of life, significant property damage, or damage to the environment" (Knight, 2002). Data processing and storage on a remote server may be sufficient for certain non-time critical MIoT use cases where unreliable and slow response time does not harm the system (Campolo et al., 2018). However, for time-critical MIoT applications, reliability and fast response as well as cybersecurity are of paramount importance. Therefore, time-critical MIoT applications require the use of novel technologies to provide a reliable and secure service. 
Due to the advancements in data communications, by providing ultra-high data rate and high throughput and very low latency technologies, it is possible to develop 5G-powered time-critical MIoT applications that will revolutionize the healthcare sector. Remote surgery is an example of a future MIoT application powered by $5 \mathrm{G}$ technologies that is also considered a time-critical application (Lacy et al., 2019). 5G is envisioned to be a network that supports multiple services in different application domains, taking into account different performance and service requirements, e.g., data transmission rate and latency (Foukas et al., 2017).

Network slicing technique is considered as the backbone of 5G technology and is an important enabler for time-critical applications equipped with wireless technologies (Campolo et al., 2017, 2018). It has been proposed to provide tailored and reliable services with limited network resources. Network slicing is able to efficiently deal with multiple tenants, some of which may have specific infrastructure requirements. For example, the use case of remote surgery within the healthcare application requires extremely low latency, which can be provided by assigning a specific network slice with such a guarantee.

Contribution. There are a variety of MIoT applications that are considered time critical. These applications have different Quality of Service (QoS) requirements. In this paper, we elaborate time-critical MIoT applications in terms of QoS requirements, addressing the technique of network slicing as a potential solution and important enabler.

Structure of the Paper. Section 2 presents the background on communication technologies. Section 3 describes the performance and security challenges in time-critical MIoT applications. Network slicing to address requirements for time-critical MIoT applications is presented in Section 4. Section 5 describes a use case for remote operations supported by $5 \mathrm{G}$ network slicing. Finally, Section 6 concludes the paper.

\section{COMMUNICATION TECHNOLOGIES}

There are a variety of existing and emerging communication technologies. These technologies can be divided into wired and wireless communication technologies. Wired communication technologies have an advantage in terms of security and can be used in combination with wireless technologies to connect sensors and end devices at the IoT level. Wireless communication technologies offer an unprecedented level of flexibility for system design and legacy updates (Johnston et al., 2018). By adopting wireless technologies and wireless sensor networks, there are significant benefits in reducing cables and connectors, lowering system costs, reducing system size and mass, reducing maintenance associated with inspection, reducing pin failures, improving system resilience to hazards, incorporating additional functionality, and increasing system flexibility for dynamic operations in the system.

IoT Technologies. Wireless radio technologies such as Bluetooth Low Energy (BLE), Zigbee, WiFi are widely used in healthcare applications today to connect sensors and end devices at IoT level (home or hospital). They are optimal in power consumption and favor mobility within a certain radius. With careful design, these wireless communication technologies can provide reliable and fast connectivity and can be used for time-critical MIoT applications in the field.

Cellular Technologies. Cellular technologies have evolved over the past 3 decades from 2G (second generation) to 5G (fifth generation). The functionalities of these generations have changed from audio-only communications to fluid video streaming. The network evolution from $2 \mathrm{G}$ to $5 \mathrm{G}$ is shown in Figure 1 . The $5 \mathrm{G}$ New Radio Evolution (NR) is being driven by a variety of key stakeholders from the traditional commercial wireless industry, a wide range of industries, and the non-terrestrial access ecosystem. It will be a key enabler for time-critical IoT applications in the industrial, transportation and medical sectors. The 5G NR technology promises to provide a common platform for radio access networks (RAN) to address the challenges of current and future time-critical IoT use cases and services, not only those we can imagine today, but also those we cannot yet imagine. This technology is expected to be a network that supports various performance and service requirements, such as data transfer rate (up to $10 \mathrm{~GB} / \mathrm{s}$ ), latency (theoretical latency of $1 \mathrm{~ms}$ ), multiple increases in base station capacity, and higher capacity to handle more connections simultaneously (Foukas et al., 2017). 


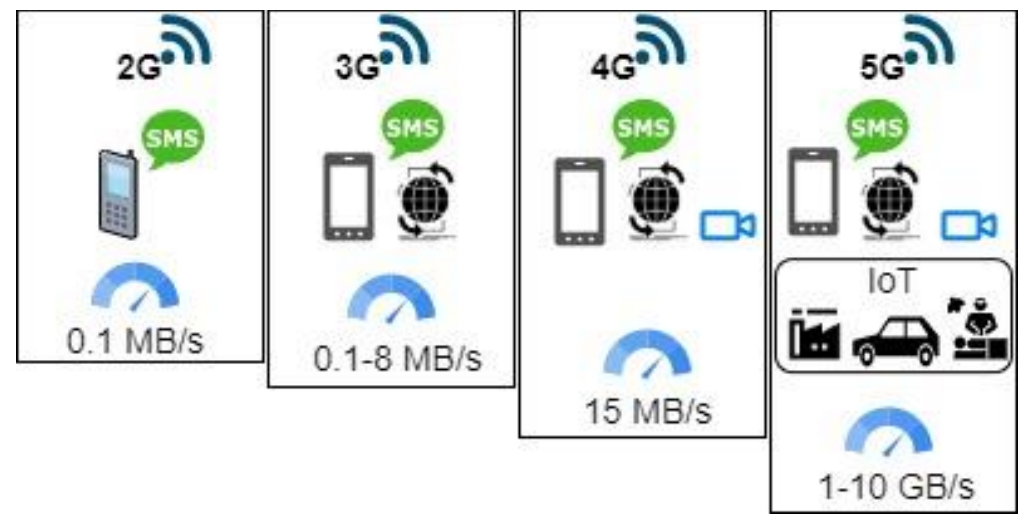

Figure 1. Overview of the evolved topology for cellular network technologies functionalities

MIoT applications can use both wired and wireless technologies, creating a heterogeneous network with different radio and software technologies. This brings challenges in the form of different requirements and constraints. There are also several critical challenges in considering wireless technologies in system design. Some examples of common challenges are unpredictable communication behavior, unreliable wireless channels, interference and collisions. Such challenges are also common in wired systems where there are cases where the medium is shared with different users. Network slicing provides the ability to efficiently manage resources while ensuring QoS of the application. The technique of network slicing is an important requirement for providing guarantees to support time-critical applications equipped with wireless technologies (Campolo et al., 2017, 2018).

\section{MIoT APPLICATIONS}

There are several performance and security challenges related to connectivity when considering time-critical MIoT applications. It requires careful design considerations and selection of connectivity technologies that can enable reliable and secure communication between a patient and a healthcare provider.

\subsection{Performance Challenges}

Reliability and fast response are paramount in case of remote surgery. Table 1 lists some of the QoS requirements for remote surgery. A packet is considered lost if it is not received by the destination application within the maximum tolerable end-to-end latency for that application. For example, $10^{-3}$ means that, on average, the application will tolerate at most 1 in 1000 packets that are not successfully received within the maximum tolerable latency. Providing ultra-high reliability close to $10^{-3}$ is defined as the maximum tolerable packet loss rate at the application layer. The 5G Ultra-Reliable Low-Latency Communications (URLLCs) service is one way to meet such a high reliability requirement.

Another challenge to enabling remote surgery is coping with resource management in data communications, although it is very possible that this could be supported by a cellular network. Traditional resource management schemes for radio access networks (typical cellular networks) consist of distributing the same radio access to all devices. This scheme may not meet the latency requirements in a remote surgery (e.g., <10ms in the case of haptic feedback). The provision of end-to-end latency under <10ms is defined as the contribution of the wireless network to the time between the sending of a packet by the source and the reception of the packet by the destination. Knowing the urgency of some services within an application, intelligent resource management is required to meet time-critical demands. For example, it is necessary to allocate more resources with dedicated channel bandwidth for such critical applications. Large delays in the network have a significant impact on the quality of surgery. It is naive to allocate the same resources to different applications, similar to traditional resource management methods. An intelligent network slicing is able to detect the requirements and constraints of the applications to allocate the resources dynamically. Therefore, it is important to develop end-to-end network slicing to manage and control the network and ensure sufficient quality of service for time-critical applications such as remote surgery. 
Table 1. QoS requirements for a remote surgery application (Perez et al., 2016; Eid et al., 2010; Cizmeci et al., 2017),(Patel \& Wang, 2010),(Hachisu \& Kajimoto, 2016)

\begin{tabular}{|l|c|c|c|c|c|}
\hline & Data Type & Latency & Jitter & Packet Loss Rate & Data Rate \\
\hline \multirow{5}{*}{ Real-time multimedia stream } & 2D camera flow & $<150 \mathrm{~ms}$ & $3-30 \mathrm{~ms}$ & $<10^{-3}$ & $<10 \mathrm{Mbps}$ \\
\cline { 2 - 6 } & 3D camera flow & $<150 \mathrm{~ms}$ & $3-30 \mathrm{~ms}$ & $<10^{-3}$ & $137 \mathrm{Mbps}-1.6 \mathrm{Gbps}$ \\
\cline { 2 - 6 } & Audio flow & $<150 \mathrm{~ms}$ & $<30 \mathrm{~ms}$ & $<10^{-3}$ & $22-200 \mathrm{Kbps}$ \\
\hline \multirow{5}{*}{ Physical vital signs } & Temperature & $<250 \mathrm{~ms}$ & - & $<10^{-3}$ & $<10 \mathrm{kbps}$ \\
\cline { 2 - 6 } & Blood pressure & $<250 \mathrm{~ms}$ & - & $<10^{-3}$ & $<10 \mathrm{kbps}$ \\
\cline { 2 - 6 } & Heart rate & $<250 \mathrm{~ms}$ & - & $<10^{-3}$ & $<10 \mathrm{kbps}$ \\
\cline { 2 - 6 } & Respiration rate & $<250 \mathrm{~ms}$ & - & $<10^{-3}$ & $<10 \mathrm{Kbps}$ \\
\cline { 2 - 6 } & ECG & $<250 \mathrm{~ms}$ & - & $<10^{-3}$ & $72 \mathrm{kbps}$ \\
\cline { 2 - 6 } & EEG & $<250 \mathrm{~ms}$ & - & $<10^{-3}$ & $86.4 \mathrm{kbps}$ \\
\cline { 2 - 6 } & EMG & $<250 \mathrm{~ms}$ & - & $<10^{-3}$ & $1.536 \mathrm{Mbps}$ \\
\hline \multirow{4}{*}{ Haptic feedback } & Force & $3-10 \mathrm{~ms}$ & $<2 \mathrm{~ms}$ & $<10^{-3}$ & $128-400 \mathrm{Kbps}$ \\
\cline { 2 - 6 } & Vibration & $<5.5 \mathrm{~ms}$ & $<2 \mathrm{~ms}$ & $<10^{-3}$ & $128-400 \mathrm{Kbps}$ \\
\hline
\end{tabular}

\subsection{Security Challenges}

In general, the integration of IoT devices and cloud services enabled by cellular technologies leads to a more complicated and dynamic threat landscape (Ahmad et al., 2017). The security threat vectors in $5 \mathrm{G}$ will be even greater than in previous generations (Madhusanka et al., 2018), even though the security of 5G mobile technology is better than that of previous ones. Therefore, attackers will have a higher chance to perform malicious activities on time-critical MIoT applications (Ahmad et al., 2017). For example, when performing a remote surgery, attackers can perform a denial of service (DoS) attack to disrupt the operation, compromising patient safety.

Therefore, it is of utmost importance to mitigate such security concerns in time-critical MIoT applications, and there are a few options to choose when a customer intends to deploy a $5 \mathrm{G}$ network, each of which has security adaptation implications. The first option is to deploy a private $5 \mathrm{G}$ network on premises and apply security measures to the private $5 \mathrm{G}$ network, but this requires investment in a separate network infrastructure. The second option is to extend the macro 5G service using Distributed Antenna Systems (DAS) (Kim et al., 2020) or alternatively small cells ${ }^{1}$, but with this option it is difficult to implement custom security solutions for a particular application. The third option is to purchase a network slice from an operator and then apply customized security solutions for a specific application. Network slicing can reduce the threat vector through isolation and enable the implementation of specific security solutions for a particular time-critical application.

\section{NETWORK SLICING}

This section highlights the idea of network slicing, which is defined within the $5 \mathrm{G}$ architecture. The term network slicing stands for "a logical network built on a common physical infrastructure that includes RAN, core networks (CNs), edge and cloud computing nodes" (Khan et al., 2020). To provide tailored and reliable services using limited network resources, Network Slicing has been proposed by the mobile industry as the key to convergence of network services (Rost et al., 2016), (Jiang et al., 2016). Network slicing can offer radio, cloud and network resources to application providers or different vertical segments without physical network infrastructure, enabling service differentiation by customizing network operations. 


\subsection{Network Slicing Enablers}

Software-defined networking (SDN) and network function virtualization (NFV) are important components for implementing network slicing. These two are the software and hardware enablers respectively in the context of $5 \mathrm{G}$.

SDN approach brings intelligence and flexibility to the network by enabling orchestration and centralized management (Kreutz et al., 2014). SDN has been defined as the physical separation of the network control plane from the forwarding plane ${ }^{2}$. This separation of the data plane from the control plane gives the SDN controller a global view of the network and provides the ability to make rapid changes to the network. More specifically, SDN creates a virtual control plane that makes intelligent decisions. In addition, the SDN controller brings increased processing power and provides the ability to run complex deep learning and machine learning algorithms to improve network performance. This is where the communication network and artificial intelligence techniques meet to improve network performance.

NFV is the virtualization of network functions traditionally executed on proprietary hardware, i.e., NFV allows network functions to be executed on standard hardware instead of dedicated hardware. It provides for instantiation of virtual network functions on hardware (Yi et al., 2018). Changes to the functions are made at the Internet Service Provider (ISP) level, as opposed to the traditional process of configuration at the customer premises. NFV can ensure that resource provisioning is optimized while meeting quality of service.

\subsection{Network Slicing Services}

According to International Telecommunication Union Radiocommunication Sector (ITU-R), which manages radio-frequency spectrum, the main objective is to support three generic services with vastly heterogeneous requirements: (i) Enhanced Mobile Broadband (eMBB), (ii) Massive Machine Type Communications (mMTC), and (iii) Ultra-Reliable Low-Latency Communications (URLLC) (Popovski et al., 2018). eMBB supports stable connections with very high peak data rates as well as moderate rates for cell edge users. mMTC supports massive numbers of IoT devices that are sporadically active and send small data payloads. URLLC supports low latency transmissions of small payload data with very high reliability. MIoT applications may require any of the above $5 \mathrm{G}$ services depending on system-level and use case-level requirements, but timecritical MIoT applications such as remote surgery require URLLC to meet their high reliability and ultra-low latency requirements.

\subsection{Network Slicing Layers}

A generic framework for network slicing (see Figure 2) considers a 3-layer model divided into (1) infrastructure, (2) control (network function), and (3) business (service) layers (Soenen et al., 2017; Foukas et al., 2017). The infrastructure layer contains all the physical components of an operator. Under this layer we can find RAN, computational and storage resources and wired connections. The control layer is a library of virtual network functions. These functions are used to control the infrastructure to establish logical networks that meet the requirements of the slice. virtual network functions are required to reserve segments of spectrum from RAN to prioritize traffic. This layer also manages and orchestrates virtual network functions. The business layer contains services and use cases, such as healthcare and intelligent transportation systems. One of the important issues in the service layer is how to map the components to the different layers of the architecture to meet the end-to-end requirements. Two types of mapping are (1) mapping service requirements to network functions and (2) mapping network functions to vendor implementations (Zhou et al., 2016).

\footnotetext{
2 https://www.opennetworking.org/
} 


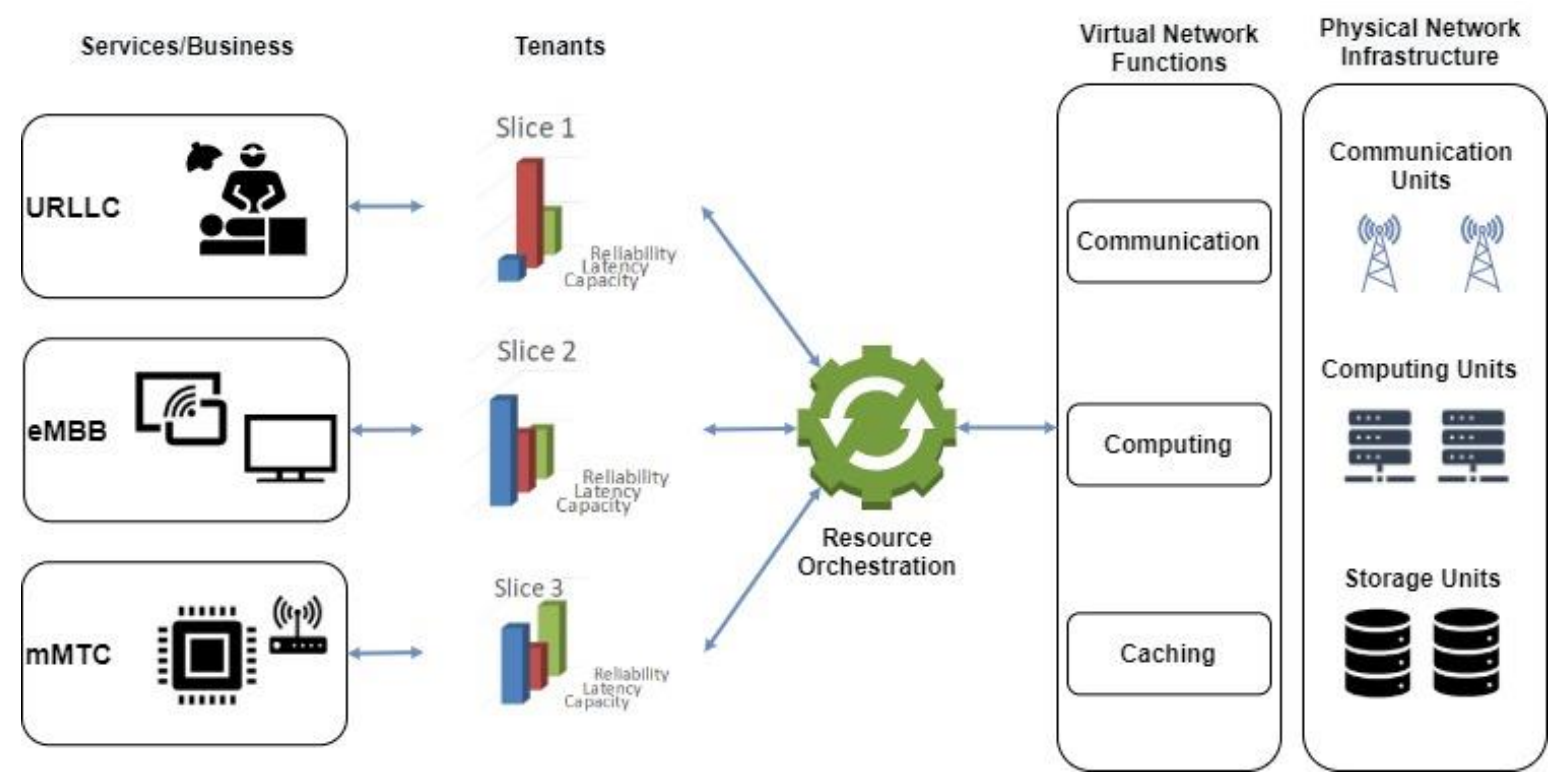

Figure 2. Generic 5G network slicing framework (Shen et al., 2020)

\subsection{Performance in Network Slicing}

Mobile network operators (MNOs) are actively working with the medical domain ${ }^{3}$ and they will be able to deploy multiple slices on their network infrastructure by splitting a physical network into multiple logical networks, where these logical networks can support customised on-demand services for different applications with different requirements and specifications. In other words, if a customer in the medical field requires 5G network infrastructure for an ultra-low latency application, an operator can provision a segment of spectrum on the RANs and a segment of bandwidth on the links in the CN connecting those RANs that can meet the demand of the customer traffic. This makes it possible to reduce network congestion that would have resulted from a shared network infrastructure. This means that the ultra-low latency $(<10 \mathrm{~ms})$ required by the remote surgery can be provided by assigning a dedicated network slice with such a guarantee. Moreover, resource management schemes in typical cellular networks are supposed to distribute RAN resources across all services. This potentially leads to increased and fluctuating delays, which is an undesirable property in time-critical applications. Intelligent network slicing can identify the QoS requirements of an application and can guarantee the latency requirements by dynamically and efficiently allocating dedicated resources for a time-critical application using intelligent resource management (Zhang et al., 2017).

\subsection{Cybersecurity in Network Slicing}

Network slicing can operate with different security functionality, such as firewall configurations, access policies, packet inspection, and can provide authentication schemes. In another word, a network slice enables applying a customized security mechanisms and policies for time-critical applications such as remote surgery contrary to using a private $5 \mathrm{G}$ network or extended macro $5 \mathrm{G}$ service using DAS. But most importantly, network slicing provides isolation between the slices ${ }^{4}$, both in terms of traffic and resources which plays an important role in protecting time-critical applications, e.g. from DoS attack. The more network slices introduced for a use case application the better the resources can be isolated which in turn improves security guard of the use case application against cyber attacks.

${ }^{3}$ https://www.groundalerts.com/5g-infrastructure-in-healthcare-market-to-witness-robust-expansionthroughout-the-forecast-period-20202025

${ }^{4} \mathrm{https} / / /$ www.ericsson.com/en/blog/2019/9/future-network-slicing-security-iot 


\section{REMOTE SURGERY}

Remote surgery is a surgery where a surgeon performs surgery on a patient that is physically in a different location as shown in Figure 3. The telesurgical system in remote surgery is a close-loop communication system consisting of forward link and feedback link. Forward link transports real-time commands in order to control the motions and rotation of robotic arms at the teleoperator, along with voice stream from the surgeon to communicate with the surgical team remotely. Feedback link transports real-time multi-modal sensory feedback from the teleoperator, including 3D video stream, force feedback e.g., pressure, and tactile feedback e.g. tissue mechanical properties, and patient's physiological data e.g. blood pressure and heart rate, along with voice stream from assistant nurses, anaesthetist and other collaborating surgeons at the patient's side.

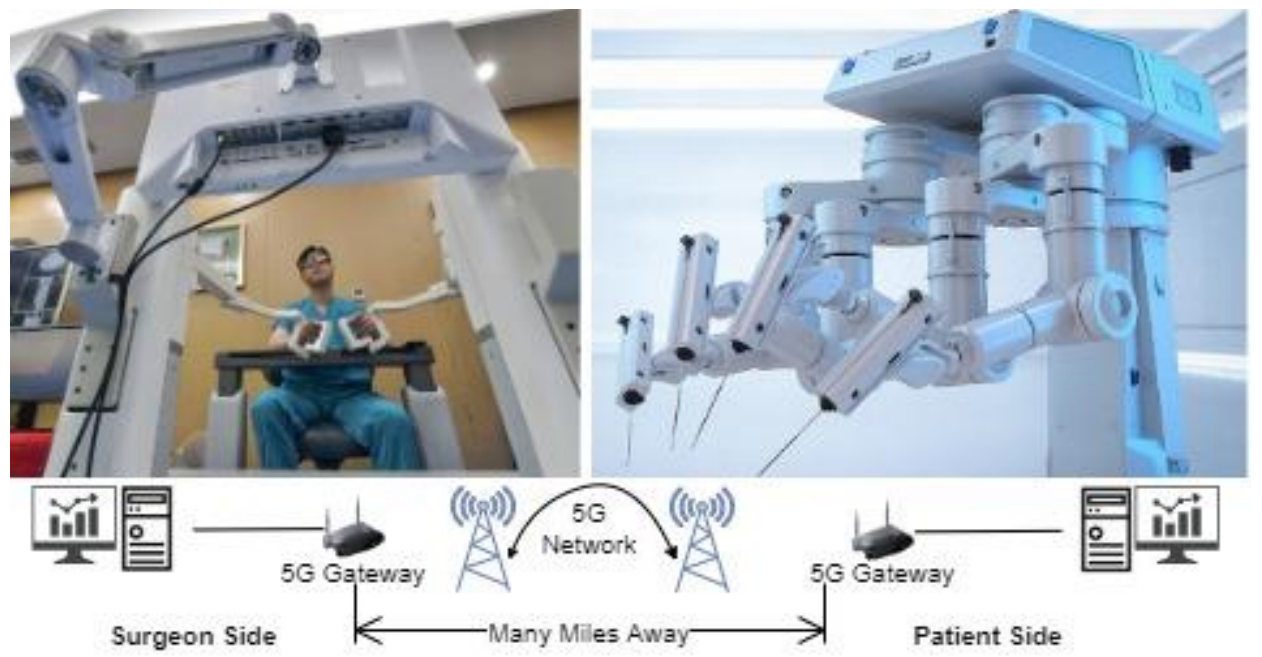

Figure 3. Remote surgery setup scenario $9,8,7$

The first remote surgery test via a $5 \mathrm{G}$ network was conducted by Ericsson and its partners ${ }^{5}$. This surgery combined haptic sensing and a transluminal surgical system, network slicing, edge computing, low latency and large bandwidth for intelligent sensing and human-machine interaction. The demonstration of remote surgery leveraged end-to-end network slicing to ensure quality of service. In this demonstration, a probe is a robotic representation of a biological finger that gives sense of touch in an invasive surgery. It can send accurate real-time localization of hard nodules in soft tissue. With 5G connectivity, intelligent network slicing separates and prioritizes time-critical functions, such as machine communication, which is required for a remote surgery.

In March 2019, People's Liberation Army General Hospital (PLAGH) carried out a real surgery on the brain of a Parkinson's disease patient in Beijing from the PLAGH Hainan Hospital 3,000km away ${ }^{6}$. 5G technologies have provided the basis and infrastructure to perform remote surgery, while achieving application requirements. Edge computing architecture has enabled local processing of big data in an edge server, and network slicing has assigned dedicated channel bandwidth to gain high throughput.

In Milan, around mid of 2020, Italian healthcare professionals were able to perform a remote surgery on the vocal cords of an adult human cadaver via a $5 \mathrm{G}$ powered surgical robotic system located at nine miles away $^{78}$. This was possible due to the fast and reliable network connections provided by $5 \mathrm{G}$ (Acemoglu et al., 2020). In Sept 2020, a similar remote surgery was conducted in East China's Shandong province using the reliable and low latency connection provided by $5 \mathrm{G}$ technology. In this procedure a patient underwent the urological surgery located in different location, Southwest China's Guizhou province ${ }^{9}$. These recent developments are clear evidence that $5 \mathrm{G}$ and its enablers are the future communication infrastructure to enable time-critical MIoT applications.

\footnotetext{
5 King's College London, Tianjin University, China Mobile Research Institute, Industrial Research Institute and CMCC Tianjin.

$6 \mathrm{https}: / / \mathrm{www} . \mathrm{medicaldevice-network.com/features/5g-remote-surgery/}$

$7 \mathrm{https}$ ://www.courthousenews.com/socially-distant-surgery-doctor-uses-5g-to-perform-remote-operation/

$8 \mathrm{https} / / /$ mytechdecisions.com/it-infrastructure/research-5g-could-usher-in-telesurgery-and-remote-surgicalprocedures/

9 https://www.chinadaily.com.cn/a/202009/25/WS5f6d6286a31024ad0ba7bd6e.html
} 


\section{CONCLUDING REMARKS}

This paper has addressed possible solutions and evolution towards enabling time-critical MIoT applications powered by $5 \mathrm{G}$ technology, namely network slicing. The e2e latency and reliability required by time-critical MIoT applications can be provided by employing 5G network slicing. However, to date reported prototypes are lacking a practical implementation of an intelligent slicing across administrative domains. Static over-provisioning of latency and bandwidth is not enough to guarantee network characteristics. The dynamic slicing of the network needs to be supported with artificial intelligence-based management system for a large network.

Network slicing minimize DoS attack by providing an isolated network and creates possibility to apply specific security solutions targeting certain time-critical application. However, the challenges of providing reliable and protected slices will grow due to the increasing threat vector as well as due to enablers like SDN and NFV (Cunha et al., 2019). The challenges will be even more if resources are allowed to be shared by multiple tenants. Therefore, network slicing shall address such challenges, e.g. compromise of a NFVs, side-channels attacks, or dealing with end-devices vulnerabilities ${ }^{10}$.

\section{ACKNOWLEDGEMENT}

This work was supported by the Swedish Knowledge Foundation (KKS) through the ARRAY project and the FlexiHealth Prospekt, and the EU Celtic Next/Vinnova project, Health5G (Future eHealth powered by 5G).

\section{REFERENCES}

Acemoglu, A., Peretti, G., Trimarchi, M., Hysenbelli, J., Krieglstein, J., Geraldes, A., ... Mattos, L. (2020, 07). Operating from a distance: Robotic vocal cord 5g telesurgery on a cadaver. Annals of Internal Medicine, 173.

Ahmad, I., Kumar, T., Liyanage, M., Okwuibe, J., Ylianttila, M., \& Gurtov, A. (2017). 5g security: Analysis of threats and solutions. In 2017 ieee conference on standards for communications and networking (cscn) (p. 193-199).

Campolo, C., Molinaro, A., Iera, A., Fontes, R. R., \& Rothenberg, C. E. (2018). Towards 5 g network slicing for the v2x ecosystem. In 2018 4th ieee conference on network softwarization and workshops (netsoft) (pp. 400-405).

Campolo, C., Molinaro, A., Iera, A., \& Menichella, F. (2017). $5 \mathrm{~g}$ network slicing for vehicle-toeverything services. IEEE Wireless Communications, 24(6), 38-45.

Cizmeci, B., Xu, X., Chaudhari, R., Bachhuber, C., Alt, N., \& Steinbach, E. (2017). A multiplexing scheme for multimodal teleoperation. ACM Transactions on Multimedia Computing, Communications, and Applications (TOMM), 13(2), $1-28$.

Cunha, V. A., da Silva, E., de Carvalho, M. B., Corujo, D., Barraca, J. P., Gomes, D., ... Aguiar, R. L. (2019). Network slicing security: Challenges and directions. Internet Technology Letters, 2(5), e125. Retrieved from https://onlinelibrary.wiley.com/doi/abs/10.1002/itl2.125

Dimitrov, D. V. (2016). Medical internet of things and big data in healthcare. Healthcare informatics research, 22(3), 156-163.

Eid, M., Cha, J., \& El Saddik, A. (2010). Admux: An adaptive multiplexer for haptic-audio-visual data communication. IEEE Transactions on Instrumentation and Measurement, 60(1), 21-31.

Foukas, X., Patounas, G., Elmokashfi, A., \& Marina, M. K. (2017). Network slicing in 5g: Survey and challenges. IEEE Communications Magazine, 55(5), 94-100.

Hachisu, T., \& Kajimoto, H. (2016). Vibration feedback latency affects material perception during rod tapping interactions. IEEE transactions on haptics, 10(2), 288-295.

Haghi, M., Neubert, S., Geissler, A., Fleischer, H., Stoll, N., Stoll, R., \& Thurow, K. (2020). A flexible and pervasive iot-based healthcare platform for physiological and environmental parameters monitoring. IEEE Internet of Things Journal, 7(6), 5628-5647.

Jiang, M., Condoluci, M., \& Mahmoodi, T. (2016). Network slicing management \& prioritization in 5g mobile systems. In European wireless 2016; 22th european wireless conference (pp. 1-6).

10 https://www.ngmn.org/publications/5g-security-recommendations-package-2-network-slicing.html 
Johnston, A. B., Schiffers, W., \& Kharaz, A. H. (2018, aug). Towards wireless technology for safety critical systems. Journal of Physics: Conference Series, 1065, 072032.

Khan, L. U., Yaqoob, I., Tran, N. H., Han, Z., \& Hong, C. S. (2020). Network slicing: Recent advances, taxonomy, requirements, and open research challenges. IEEE Access, 8, 36009-36028.

Kim, J., Sung, M., Cho, S., Won, Y., Lim, B., Pyun, S., ... Lee, J. H. (2020). Mimo-supporting radio-over-fiber system and its application in mmwave-based indoor 5g mobile network. Journal of Lightwave Technology, 38(1), 101-111.

Knight, J. C. (2002). Safety critical systems: challenges and directions. In Proceedings of the 24th international conference on software engineering (pp. 547-550).

Kreutz, D., Ramos, F. M., Verissimo, P. E., Rothenberg, C. E., Azodolmolky, S., \& Uhlig, S. (2014). Software-defined networking: A comprehensive survey. Proceedings of the IEEE, 103(1), 14-76.

Lacy, A., Bravo, R., Otero-Piñ eiro, A., Pena, R., De Lacy, F., Menchaca, R., \& Balibrea, J. (2019). 5g-assisted telementored surgery. British Journal of Surgery.

Madhusanka, L., Ijaz, A., Bux, A. A., Andrei, G., \& Mika, Y. (2018). A comprehensive guide to $5 g$ security. https://doi.org/10.1002/9781119293071: John Wiley \& Sons, Incorporated.

Patel, M., \& Wang, J. (2010). Applications, challenges, and prospective in emerging body area networking technologies. IEEE Wireless communications, 17(1), 80-88.

Perez, M., Xu, S., Chauhan, S., Tanaka, A., Simpson, K., Abdul-Muhsin, H., \& Smith, R. (2016). Impact of delay on telesurgical performance: study on the robotic simulator dv-trainer. International journal of computer assisted radiology and surgery, 11(4), 581-587.

Popovski, P., Trillingsgaard, K. F., Simeone, O., \& Durisi, G. (2018). 5g wireless network slicing for embb, urllc, and mmtc: A communication-theoretic view. IEEE Access, 6, 55765-55779.

Rees, P., van der Gaag, N., de Beer, J., \& Heins, F. (2012). European regional populations: Current trends, future pathways, and policy options. European Journal of Population / Revue europ'eenne de D'emographie, 28.

Rost, P., Banchs, A., Berberana, I., Breitbach, M., Doll, M., Droste, H., ... Sayadi, B. (2016). Mobile network architecture evolution toward 5g. IEEE Communications Magazine, 54(5), 84-91.

Shen, X., Gao, J., Wu, W., Lyu, K., Li, M., Zhuang, W., ... Rao, J. (2020). Ai-assisted networkslicing based next-generation wireless networks. IEEE Open Journal of Vehicular Technology, 1, 45-66.

Soenen, T., Banerjee, R., Tavernier, W., Colle, D., \& Pickavet, M. (2017). Demystifying network slicing: From theory to practice. In 2017 ifip/ieee symposium on integrated network and service management (im) (pp. 1115-1120).

Yi, B., Wang, X., Li, K., Huang, M., et al. (2018). A comprehensive survey of network function virtualization. Computer Networks, 133, 212-262.

Zhang, H., Liu, N., Chu, X., Long, K., Aghvami, A.-H., \& Leung, V. C. (2017). Network slicing based 5g and future mobile networks: mobility, resource management, and challenges. IEEE communications magazine, 55(8), 138-145.

Zhou, X., Li, R., Chen, T., \& Zhang, H. (2016). Network slicing as a service: enabling enterprises' own software-defined cellular networks. IEEE Communications Magazine, 54(7), 146-153. 